Check for updates

\section{London}

Cite this as: BMJ 2021;372:n308 http://dx.doi.org/10.1136/bmj.n308 Published: 02 February 2021

\title{
Covid-19: People who have had infection might only need one dose of $\mathrm{mRNA}$ vaccine
}

\section{Jacqui Wise}

People who have already had confirmed covid-19 might only need a single dose of a mRNA vaccine, two small studies have indicated.

The first study, which was published as a preprint and has not yet been peer reviewed, found that seropositive individuals had a rapid antibody response after one dose of either the Pfizer-BioNTech or Moderna vaccines. ${ }^{1}$ It also found that people who had previously had covid-19 reported side effects more frequently after the first dose-in line with the level reported for the second dose in phase III vaccine trials.

The researchers, from Mount Sinai in New York and Paris, examined the antibody responses in 109 people -68 of them had never had covid-19, whereas 41 had previously tested positive. The antibody titres of those who had previously had covid-19 were 10-20 times those of people who had never had it at the same time points. Two weeks after a single dose of vaccine, people who had previously had covid-19 had antibody concentrations that were as high, or up to 10 times higher, than the levels seen in uninfected people who had received two doses of the vaccine.

The researchers said that ongoing follow-up studies would show whether these early differences in immune response were maintained over time. They wrote that giving people who had been previously infected with SARS-CoV-2 just one dose of vaccine "would not negatively impact on their antibody titres, [would] spare them from unnecessary pain, and [would] free up many urgently needed doses.”

The study also compared the frequency of adverse reactions after the first dose of vaccine in 231 people including 83 who had previously tested positive for covid-19. Localised injection site symptoms such as pain or swelling were similar in both groups of patients. But those with pre-existing immunity experienced systemic side effects such as fatigue, headache, chills, fever, and muscle or joint pains with considerably higher frequency.

A second small study, also published as a preprint, reported similar findings. ${ }^{2}$ This study, from the University of Maryland, looked at antibody responses to a single dose of the Pfizer-BioNTech or Moderna vaccines in 59 healthcare workers. Those who had previously been infected with SARS-CoV-2 had a clear antibody response, which peaked at 10 and 14 days after vaccination. At all time points, healthcare workers with previous infection showed statistically significantly higher antibody levels than those who hadn't been infected.

Eleanor Riley, professor of immunology and infectious disease at the University of Edinburgh, commented: "The authors of both papers suggest that people who have had a PCR confirmed covid-19 infection may only require one dose of the vaccine. Certainly, this would appear to provide them with protection that is at least as good as two doses of vaccine. However, incorporating this into a mass vaccination programme may be logistically complex and it may be safer, overall, to ensure that everyone gets two doses."

And Lawrence Young, virologist and professor of molecular oncology, at the University of Warwick, said: "If future work can confirm this high level of immunity post a single mRNA vaccine in this group of individuals, this could become a viable option when there are concerns around vaccine supply."

Krammer F, Srivastava K. PARIS team, Simon V. Robust spike antibody responses and increased reactogenicity in seropositive individuals after a single dose of SARS-CoV-2 mRNA vaccine. MedRxiv [Preprint]. 2021. https://www.medrxiv.org/content/10.1101/2021.01.29.21250653v1

2 Saadat S, Rikhtegaran-Tehrani Z, Logue J, et al. Single dose vaccination in healthcare workers previously infected with SARS-CoV-2. MedRxiv [Preprint]. 2021. https://www.medrxiv.org/content/10.1101/2021.01.30.21250843v2

This article is made freely available for use in accordance with BMJ's website terms and conditions for the duration of the covid-19 pandemic or until otherwise determined by BMJ. You may use, download and print the article for any lawful, non-commercial purpose (including text and data mining) provided that all copyright notices and trade marks are retained. 